\title{
QUALIDADE DA INFORMAÇÃO COM APLICAÇÃO DO DESIGN THINKING
}

Almir dos Santos Albuquerque ${ }^{1}$, Rogério Cid Bastos ${ }^{2}$, Silvio Serafim da Luz Filho ${ }^{2}$

'Doutorando no Programa de Pós-Graduação em Engenharia e Gestão do Conhecimento (EGC). Universidade Federal de Santa Catarina (UFSC), Florianópolis, Brasil. E-mail: asaalbuquerque@gmail.com

${ }^{2}$ Professor Doutor do Programa de Pós-Graduação em Engenharia e Gestão do Conhecimento (EGC). Universidade Federal de Santa Catarina (UFSC), Florianópolis, Brasil.

Recebido em: 15/02/2020 - Aprovado em: 15/03/2020 - Publicado em: 30/03/2020 DOI: 10.18677/EnciBio_2020A20

\begin{abstract}
RESUMO
rápido crescimento do volume de informações disponibilizadas na web e a ausência de padrões nessas informações, tornaram difíceis para os usuários distinguir quais informações são precisas e confiáveis, ou seja, quais informações possuem ou não, qualidade. As diversas informações acessadas pelos usuários precisam ter critérios que garantam o mínimo de qualidade. Os designers estão sempre envolvidos nos desafios de criar um novo produto, serviço, comunicação ou marca, que atendam ao desejo do consumidor ou usuário de forma inédita e que the confira uma identidade distintiva. O Design Thinking pode ser utilizado como uma das formas de vencer esses desafios, em especial, na busca por inovação e desenvolvimentos de novos produtos. Neste artigo as fases de imersão, análise e síntese, ideação e prototipação, oriundas do Design Thinking, foram aplicadas ao produto informação, com intuito de disponibilizar a informação com utilidade, credibilidade e qualidade em sites web para os usuários, e restou evidenciado a viabilidade da utilização desta técnica.
\end{abstract}

PALAVRAS-CHAVE: design thinking, informação, qualidade.

INFORMATION QUALITY WITH APPLICATION OF DESIGN THINKING.

\begin{abstract}
The rapid growth in the volume of information made available on the web and the absence of standards in this information, made it difficult for users to distinguish which information is accurate and reliable, that is, which information has or does not have quality. The various information accessed by users must have criteria that guarantee the minimum quality. Designers are always involved in the challenges of creating a new product, service, communication or brand, meeting the desire of the consumer or user in an unprecedented way and giving it a distinctive identity. Design Thinking can be used as one of the ways to overcome these challenges, especially in the search for innovation and new product developments. In this article, the phases of immersion, analysis and synthesis, ideation and prototyping, originating from Design Thinking, were applied to the product information, in order to provide the information with usefulness, credibility and quality on websites for users, where the viability remained using this technique.
\end{abstract}

KEYWORDS: design thinking, information, quality. 


\section{INTRODUÇÃO}

As Tecnologias da Informação e Comunicação (TIC's) adentraram os lares e se estabeleceram muito rapidamente. Computadores e smartfones (telefone celular com múltiplas funções), deixaram de ser utensílios de luxo e tornaram-se um "simples eletrodoméstico" (CAVALCANTE, 2018). Estimulado por fatores sociais, econômicos e tecnológicos, o cidadão em geral, está cada vez mais, acessando e utilizando o ciberespaço que lhe é disponibilizado, tornando-se assim, um grande leitor e consumidor de informação (OLIVEIRA; JORENTE, 2019).

Destarte, a web tornou-se uma das principais fontes informacionais utilizadas pelos usuários. Com o acúmulo exponencial de informações disponíveis e com o rápido desenvolvimento dos processos tecnológicos de registro e de acesso a essas informações, o cidadão passou a conviver com um problema fundamental que é o de selecionar no imenso estoque de informações atualmente existente, aquelas que têm qualidade e utilidade para ele (CAVALCANTE, 2018; OLIVEIRA; JORENTE, 2019).

A qualidade da informação é considerada como um conceito multidimensional, com diferentes características atribuídas dependendo do ponto de vista filosófico do autor, em que vários fatores determinam o seu estado. Essa qualidade, é baseada nas dimensões e atributos das fontes de informação e no papel desempenhado pelos usuários consumidores de informação (ALVARES; GONÇALVES, 2017).

As formas de produção e transmissão da informação, vem alterando a dinâmica do setor de serviços ao longo dos anos. Essas transformações explicitaram a necessidade das organizações darem um tratamento gerencial à documentação $\mathrm{e}$ à informação evitando o acúmulo e o desperdício. Nesse raciocínio, a informação e - conhecimento são insumos para desenvolver novos produtos, materiais e processos, potencializando o desenvolvimento organizacional, por meio da tomada de decisão, de modo a gerar diferenciais competitivos (NASCIMENTO et al., 2016; MESQUITA; FARIAS, 2018).

As abordagens da qualidade da informação baseada no produto enfatiza a informação como coisa, atribuindo-se à qualidade da informação atributos ou dimensões, tais como: Abrangência, relacionada ao valor intrínseco da informação; Acessibilidade, importância em termos de redução do tempo necessário ao acesso à informação; Atualidade, referindo-se à consonância com o ritmo de produção da informação, opondo-se à obsolescência; Confiabilidade, em termos da credibilidade no conteúdo e na fonte da informação; Precisão, sentido aproximado de exatidão, correção, remetendo à forma de registro fiel ao fato que representa e Validade, pressupondo integridade da fonte de informação e forma de registro fiel ao fato que representa (CAVALCANTE, 2018).

A informação pode ser visualizada como "produto informação", que é uma analogia com produtos resultantes de processos de manufatura tradicional (ALBUQUERQUE et al., 2009). Esses produtos "físicos" são resultantes de um processo de produção onde matérias primas são processadas. O "produto informação" (bem intangível) é resultante do processamento de dados (brutos) por sistemas de informação (CONQUE FILHO; FAVARETTO, 2009).

As diversas informações acessadas pelos cidadãos precisam ter credibilidade, conteúdo, boa apresentação, acessibilidade, navegabilidade, interatividade, confiabilidade, validade, tempestividade, etc, enfim; critérios que 
garantam o mínimo de qualidade para o usuário (CAVALCANTE, 2018). Nesse contexto, com a aplicação do Design Thinking pode-se disponibilizar o Produto Informação, com qualidade para o usuário?

Design Thinking é "a disciplina que utiliza a sensibilidade e os métodos do designer para corresponder às necessidades dos consumidores, com o que é tecnologicamente factível, e o que a estratégia viável de negócio possa converter em valores para consumidores e oportunidades de mercado" (BROWN, 2008). É "uma abordagem focada no ser humano que vê na multidisciplinaridade, colaboração e tangibilização de pensamentos e processos, caminhos que levam a soluções inovadoras para negócios" (VIANNA et al., 2012). É uma ferramenta que propicia imaginar estados futuros, pensar por meio do processo de design, assim como gerar produtos, serviços e experiências reais (COOPER et al., 2010; KOLKO, 2018).

Além de que, o Design Thinking introduz novidades e revela novos significados aos produtos, serviços ou relacionamentos. É a ampliação do conceito de design, muito além da atuação na produção industrial, passando a ser aplicado em qualquer situação, tanto em objetos tangíveis como em sistemas intangíveis. Promove experiências mais inteligentes, significativas e gratificantes: No desenvolvimento de um projeto, no planejamento estratégico e na solução de problemas. Não é um processo linear, mas um exercício interativo e funciona fora da lógica (OROFINO, 2011).

A técnica utilizada na elaboração deste trabalho foi oriunda do Desing Thinking e suas ferramentas, que passam por etapas e variações de perspectivas projetistas no desenvolvimento do produto. Essas ferramentas, vêm sendo utilizadas pelas empresas em contextos de inovação e em especial, para desenvolvimento de novos produtos (RIZARDI, 2017). Dessa forma, IDEO (2015) afirma que no Design Thinking existem soluções na interseção de três fatores: desejo, factibilidade e viabilidade, ou seja, quando a solução é desejável, financeiramente viável e tecnologicamente factível, a inovação acontece já que esses fatores existem simultaneamente. Então, para que os resultados sejam atingidos é necessário focar no usuário (LIRA et al., 2019).

O objetivo deste trabalho de pesquisa é a aplicação do Design Thinking adaptado ao produto informação, visando a disponibilização da informação com qualidade em sites web, para o usuário.

\section{MATERIAIS E MÉTODOS}

Este trabalho, apresenta-se como uma pesquisa científica que distingui os avanços dos conhecimentos existentes em relação a qualidade da informação em sites web. Caracterizada como de natureza empírica, foi pautada nos conceitos encontrados na literatura específica e na documentação pesquisada, para descrever e fundamentar os principais elementos dos temas apresentados. Foi realizado no primeiro trimestre de 2019, com os alunos participantes da disciplina Design Thinking, cursada no programa de Pós Graduação em Engenharia e Gestão do Conhecimento (EGC) da universidade Federal de Santa Catarina (UFSC), como um dos critérios de avalição final do curso; cujo o objetivo foi a aplicação do Design Thinking adaptado ao produto informação, visando a disponibilização da informação com qualidade em sites web, para o usuário.

As fases de imersão, análise e síntese, ideação e, prototipação, conforme ilustrado na Figura 1; foram organizadas e adaptadas, a fim de demonstrar a aplicação dos conceitos desta ferramenta e também o modo de pensar, com 
benefícios sensíveis para as pessoas e organizações que estão em busca de inovação e desenvolvimentos de produtos.

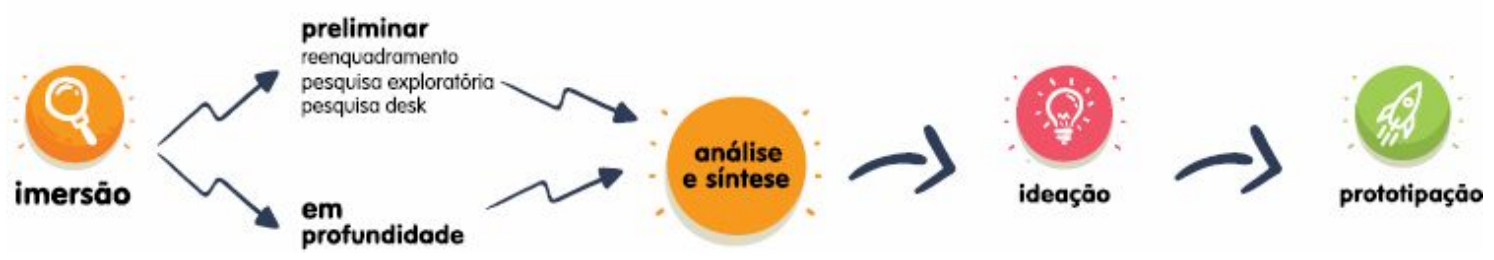

FIGURA 1: Fases do Design Thinking.

FONTE: SOUZA; MENDONÇA, (2019).

$\mathrm{Na}$ fase de imersão, foi estudado o problema sob diferentes perspectivas, a partir do levantamento, da seleção e da análise de informações. Esta foi dividida em imersão preliminar e imersão em profundidade. A imersão preliminar constituiu-se no reenquadramento do problema, com coletas iniciais de dados e conhecimentos prévios dos alunos sobre o tema proposto, seguidos de pesquisa exploratória (uma pesquisa de campo preliminar por meio de entrevistas e anotações das discussões em equipe) e pesquisa desk, por meio de consulta a fontes de dados eletrônicos (sites web em geral).

A imersão em profundidade consistiu em um mergulho a fundo no contexto (sites web) dos usuários e do assunto trabalhado. Adotando-se diferentes estratégias de pesquisa que possibilitou estudar o público-alvo e suas necessidades em um contexto real. Onde realizou-se uma organização dos dados em categorias e do volume de informações gerado.

$\mathrm{Na}$ fase de análise e síntese, várias reflexões foram realizadas, embasadas nos levantamentos efetuados nas fases de imersão preliminar e em profundidade, transformando-as em cartões post-it (papel adesivo que serve para fixar notas temporariamente sobre qualquer tipo de superfície), para que facilitar uma rápida consulta e o seu manuseio.

$\mathrm{Na}$ fase de ideação, gerou-se propostas de solução e em seguida, montou-se sessões de cocriação com as equipes de alunos. Desta forma, foi formado uma lista de ideias a serem validadas para orientar a próxima fase. Na última fase, a de prototipação, transformou-se as ideias em planos de ação e na sequência, a construção do protótipo final, sendo este, a representação conceitual que serviu como referência para a construção da ideia final. A figura 2, ilustra as etapas que foram seguidas na aplicação do Design Thinking ao produto informação. 


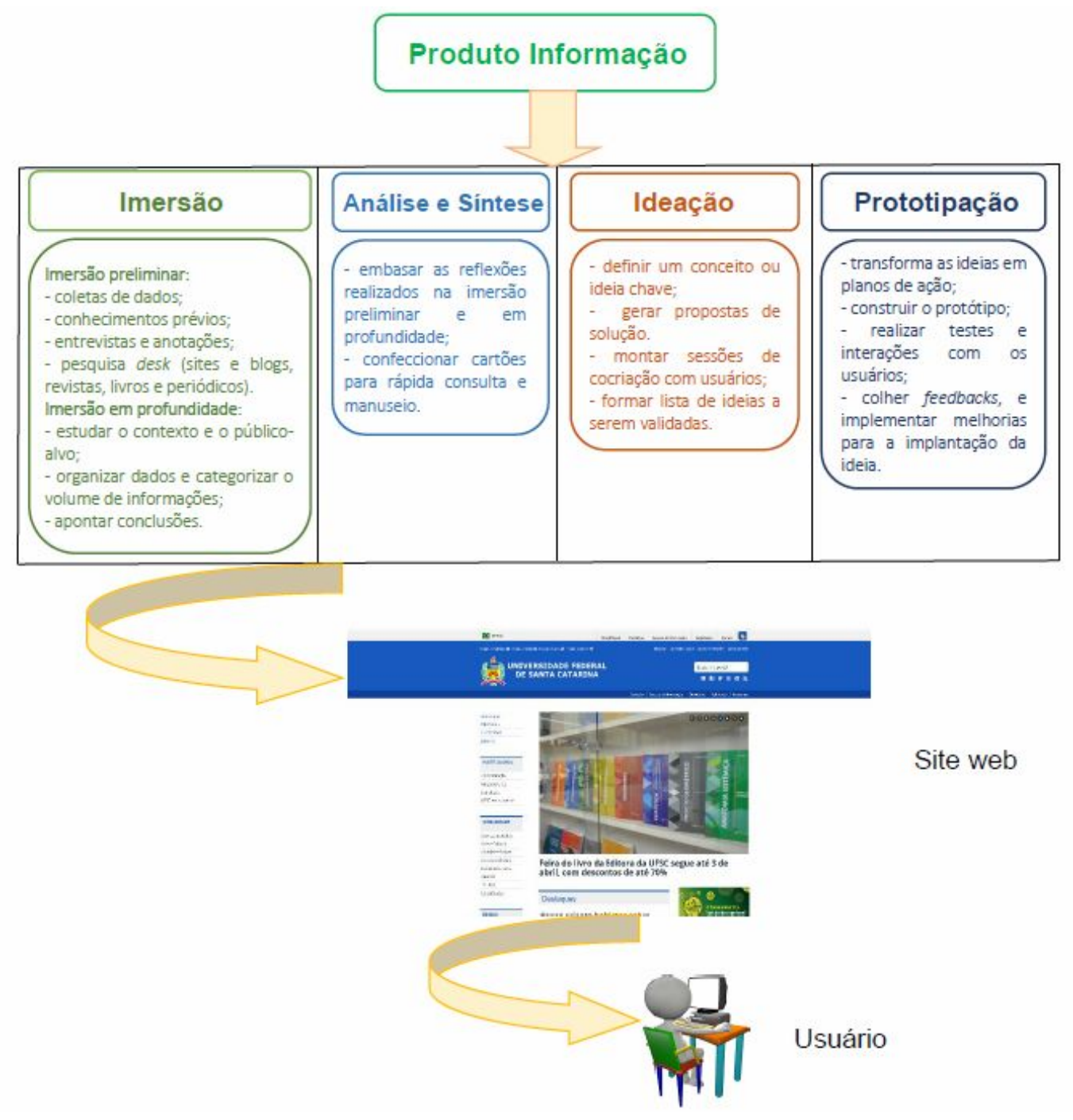

FIGURA 2 - Design Thinking aplicado ao "Produto Informação" FONTE: Próprios autores, 2020.

\section{RESULTADOS E DISCUSSÃO}

Foram efetuadas pesquisas bibliográficas, algumas aqui citadas, referentes aos temas: Qualidade da Informação e Design Thinking, a fim de auxiliar na aplicação dos conceitos e das ferramentas utilizadas nas fases do projeto descritas na figura 2.

Pode-se perceber que com o Design Thinking, bem estudado e aplicado é possível com certa facilidade e praticamente sem nenhum investimento, trazer benefícios financeiros, como também na melhoria dos processos de criação e elaboração de projetos de desenvolvimento de novos produtos, em uma organização.

O início do projeto deu-se com a primeira fase, a de imersão (preliminar e profundidade). Onde o contexto do problema foi definido e enunciado em conjunto com as equipes, a partir de discussões sobre a problemática (qualidade da informação) e anotações dos insights oriundos das observações feitas por membros das equipes. Chegando-se assim, a definição do problema e consequentemente ao seu escopo. Conforme preconizado por Vianna et al. (2012) a imersão preliminar, ENCICLOPÉDIA BIOSFERA, Centro Científico Conhecer - Jandaia-GO, v. 17 n.31; p. 211 
define o escopo do projeto e suas fronteiras, além de identificar os perfis de usuários e outros atores-chave que deverão ser abordados, sendo possível também, levantar as áreas de interesse a serem exploradas de forma a fornecer insumos para a elaboração dos temas que serão investigados na imersão em profundidade.

Incialmente, as dificuldades encontradas pelas equipes estavam relacionadas a inexperiência quanto ao uso de técnicas para gerenciamento do tempo em especial, o controle do tempo gasto nas discussões sobre a problemática. Sendo necessário a chamada de atenção para que os membros ficassem alertas quanto a utilização adequada do recurso tempo, para o desenvolvimento de cada etapa do projeto.

A partir das discussões e anotações realizadas na fase anterior, uma análise foi realizada e sintetizada com a geração de uma grande quantidade de cartões post-it de insights que foram organizados e classificados em: tecnologias, acessibilidade, atualidade, confiabilidade, precisão e validade. Essa organização fezse necessária para obter-se padrões e criar desafios que auxiliassem na compreensão do problema como recomenda Vianna et al. (2012).

A geração de ideias tende a ser o processo mais criativo ou "fora da caixa" relativamente ao entendimento mental da questão. Para auxiliar o processo criativo há alguns processos de auxílio, mas que cada pessoa pode adotar os processos que melhores resultados lhe proporcionem (RIZARDI, 2017; ROBALINHO, 2018). De acordo com Brown (2008), nesta etapa é primordial a seleção e classificação das melhores ideias com o intuito de torná-las tangíveis, evoluindo da geração para prototipagem.

O protótipo é a tangibilização de uma ideia, a passagem do abstrato para o físico de forma a representar a realidade e propiciar validações. É um instrumento de aprendizado sob dois aspectos: da ótica da equipe de projeto que dá forma à ideia, e da ótica do usuário ao interagir com o modelo criado, podendo avaliá-lo e fornecer insumos para seu aperfeiçoamento (VIANNA et al., 2012). Dessa forma, o protótipo final foi construído pela equipe e apresentado como sendo a ideia final do projeto.

\section{CONCLUSÃO}

Neste trabalho, apresentamos o Design Thinking como uma técnica que pode ser utilizada para conduzir pesquisas na área da Ciência da Informação, visando a concepção de soluções centradas naqueles usuários que estão diretamente envolvidos com a utilização e o consumo de Informação, em especial, em sites web. Esta técnica vem sendo aplicada de diversas maneiras em projeto de inovação. A cultura do design em si, tornou-se uma poderosa ferramenta estratégica para as organizações.

Identifica-se ainda o potencial de contribuição do Design Thinking no contexto de produtos intangíveis, em especial na qualidade da informação, cuja abordagem insere o usuário e suas necessidades no centro das decisões de projeto. Dessa forma, entende-se que a compreensão do indivíduo também é parte essencial no design do Produto Informação.

Além do entendimento do usuário, foi possível perceber que as ferramentas auxiliam em diversos outros aspectos do projeto, tais como: planejamento, coleta de dados, geração e seleção de ideias, prototipagem, testes, e no gerenciamento das soluções; restando assim a realização de estudos mais aprofundados de suas contribuições nestas diferentes atividades de projetos. 
A proposta de envolver tanto, clientes como usuários, nas etapas do processo de desenvolvimento das soluções é altamente recomendável para atingir resultados de fato eficientes; da definição do direcionamento à Imersão; do reposicionamento à Ideação; da Prototipação; do desenvolvimento à Implementação e o suporte do acompanhamento.

Com base na literatura específica da área, a pesquisa documental e a pesquisa aqui realizada, constata-se que a adoção das fases do Design Thinking em projetos de inovação vem sendo utilizado com sucesso. Desta forma, restou evidenciado a viabilidade da utilização do Design Thinking, através das fases de imersão, análise e síntese, ideação e prototipação; adaptadas e aplicadas ao Produto Informação, visando a disponibilização da informação em sites web, com utilidade, credibilidade e qualidade para o usuário.

\section{REFERÊNCIAS}

ALBUQUERQUE, A. S.; BASTOS, R. C.; LINO, M. R. O. Qualidade da informação em portais do judiciário. Encontros Bibli: Revista eletrônica de biblioteconomia e ciência da informação, ISSN 1518-2924, Florianópolis, v. 14, n.27, 2009. Disponível em: <https://periodicos.ufsc.br/index.php/eb/article/view/15182924.2009v14n27p115/19690>, $\quad$ DOI: $\quad$ https://doi.org/10.5007/15182924.2009v14n27p115.

ALTMAN, M.; HUANG, T. T.; BRELAND, J. Y. Design Thinking in Health Care. Preventing Chronic Disease, 2018; 15:180128. Disponível em: <https://www.cdc.gov/pcd/issues/2018/18_0128.htm>, DOI: https://doi.org/10.5888/pcd15.180128.

ALVAREZ, G. M.; GONÇALVES, A. L. Qualidade da Informação e Recuperação de Informação: uma revisão da literatura. ReTIC - Revista Tecnologia da Informação e Comunicação: Teoria e Prática, ISSN 2526-6004, Florianópolis, p. 62-76, Vol. 1, n. 1 2017. Disponível em: <http://www.portaldeperiodicos.unisul.br/index.php/ReTIC/article/view/4520>.

BROWN, T. Design Thinking. In: Harvard Business Review, p. 84-92, 2008. Disponível em: https://new-ideocom.s3.amazonaws.com/assets/files/pdfs/IDEO_HBR_DT_08.pdf.

CAVALCANTE, W. A. Credibilidade e qualidade informacional na web. In: XXX Encontro Regional de Estudantes de Biblioteconomia, Documentação, Ciência e Gestão da Informação - EREBD 2018. Disponível em: <https://www.academia.edu/38669640/XXI_Encontro Regional dos Estudantes de Biblioteconomia

documenta\%C3\%87\%C3\%830_CI\%C3\%8ANCIA_E_GEST\%C3\%830_DA_INFOR MA\%C3\%87\%C3\%83O>.

CONQUE FILHO, V. M.; FAVARETTO, F. Verificação da utilização e da importância da medição da qualidade da informação em processos de produção de produtos físicos e de informação. Revista Gestão Industrial, v. 5, n. 2, 2009. Disponível em: <https://periodicos.utfpr.edu.br/revistagi/article/view/342>. DOI: $10.3895 /$ S180804482009000200001. 
COOPER, R.; JUNGINGER, S.; LOCKWOOD, T. Design thinking and design management: A research and practice perspective. In: LOCKWOOD, T. (Ed.), Design thinking. New York, NY: Allworth Press, p. 57-64, 2010. Disponível em: <https://onlinelibrary.wiley.com/doi/abs/10.1111/j.1948-7169.2009.00007.x>. DOI: https://doi.org/10.1111/j.1948-7169.2009.00007.x.

IDEO. Design thinking for libraries: a toolkit for patron-centered design. EUA: Centered Design, 2015. Disponível em: <http://designthinkingforlibraries.com/>.

KOLKO, J.; The Divisiveness of Design Thinking. INTERACTIONS.ACM.ORG, MAYJUNE 2018. Disponível em: <https://interactions.acm.org/archive/view/may-june2018/the-divisiveness-of-design-thinking>. DOI: 10.1145/3194313.

LIRA, R. A.; CARPES, C. E. P.; DAVILA, G.; VARVAKIS, G. Thinking em Bibliotecas: evidências da Literatura. P2P E INOVAÇÃO, [S.I.], v. 6, n. 1, p. 104-116, oct. 2019. ISSN 2358-7814. Disponível em: <http://revista.ibict.br/p2p/article/view/4877>. DOI: https://doi.org/10.21721/p2p.2019v6n1.p104-116.

MELO, A; ABELHEIRA, R. Design thinking \& thinking design: metodologia, ferramentas e reflexões sobre o tema. São Paulo: Novatec, 2015.

MESQUITA, J. V. P.; FARIAS, J. S. Avaliação da qualidade da informação disponibilizada em sistemas de internet banking e mobile banking na ótica de usuários. E\&G Economia e Gestão, Belo Horizonte, v. 18, n. 49, Jan./Abr. 2018. ISSN 1984-6606.

NASCIMENTO, N. M. do; SANTOS, J. C. dos; VALENTIM, M. L. P.; MOROCABERO, M. M. O estudo das gerações e a inteligência competitiva em ambientes organizacionais. Perspectivas em Gestão \& Conhecimento, João Pessoa, v. 6, n.esp., p. 29-44, jan. 2016. Disponível em: <https://periodicos.ufpb.br/ojs2/index.php/pgc/article/view/27381>.

OLIVEIRA, J. A. D. B.; JORENTE, M. J. V. Design da Informação e sua relevância para a Ciência da Informação. Encontros Bibli: Revista eletrônica de biblioteconomia e ciência da informação, v. 24, n. 54, p. 25-37, jan./abr., 2019. ISSN 1518-2924. Disponível em: $<$ https://periodicos.ufsc.br/index.php/eb/article/view/1518-2924.2019v24n54p25>, DOI: 10.5007/1518-2924.2019v24n54p25.

OROFINO, M. A. Design Thinking e o Contexto Urbano. Apresentação realizada em 16/7/2011, no TEDxCuritiba, Brasil. Disponível em: $<$ https://pt.slideshare.net/augustaorofino/design-thinking-e-o-contexto-urbano>. Acesso em: 30 jun. 2019.

RIZARDI, B. M. DESING THINKING PARA INOVAÇÃO SOCIAL . Universidade Estadual de Londrina. 2017. Livro Digital. Disponível em: <https://goo.gl/MVofki>. Acesso em: 29 março 2019.

ROBALINHO, M. Desing Thinking in Software Development. Universidade Portucalense Infante D. Henrique. Lisboa, Portugal. 2018. Disponível em: 
$<$ https://www.researchgate.net/publication/331497974_Designing_Thinking_in_Softw are_Development>.

SOUZA, A.; MENDONÇA, A. Design thinking na publicidade. Comunicação \& Educação, v. 24, n. 1, p. 30-43, 31 maio 2019. Disponível em: <http://www.revistas.usp.br/comueduc/article/view/147448>. DOI: https://doi.org/10.11606/issn.2316-9125.v24i1p30-43.

VIANNA, M.; VIANNA, Y.; ADLER, I K.; LUCENA, B.; RUSSO, B. Desing thinking: inovação em negócios. Rio de Janeiro: MJV Press, 2012. 162p. : il., 24 cm, ISBN 978-85-65424-00-4. Disponível em: <https://www.livrodesignthinking.com.br/>. Acesso em: 29 março 2019. 